\title{
Novel Module for Plasma Wakefield Acceleration of a Positron Beam
}

\author{
X. Wang ${ }^{1}$, R.Ischebeck ${ }^{2}$, C. Joshi ${ }^{3}$, P. Muggli ${ }^{1}$, T. Katsouleas ${ }^{1}$ \\ ${ }^{1}$ University of Southern California, Los Angeles, CA 90007 \\ ${ }^{2}$ Stanford Linear Accelerator Center, Stanford, CA 94025 \\ ${ }^{3}$ University of California at Los Angeles, Los Angeles, CA 90095
}

\begin{abstract}
A novel approach for studying positron acceleration physics without an external positron source is developed. This scheme uses an $\mathrm{e}^{-}$beam to both create positrons in a target before the plasma and to excite the wake that accelerates these positrons. 2-D PIC simulations show that ultra-short $\mathrm{e}^{+}$bunches can be focused and accelerated.
\end{abstract}

Keywords: plasma wakefield, positron acceleration, positron source

PACS: $52.40 . \mathrm{Mj}$

\section{INTRODUCTION}

High-gradient acceleration of positrons and electrons are both equally important to a plasma based $\mathrm{e}^{+} / \mathrm{e}^{-}$linear collider. Compared to electron acceleration, positron acceleration has been less studied due to lack of available relativistic $\mathrm{e}^{+}$beams. In this paper we present simulation results that show that positrons generated by an ultrarelativistic electron beam in a high- $Z$ target can be trapped in, and accelerated by the wake driven by the $\mathrm{e}^{-}$beam in a subsequent plasma. The trapping occurs in a phase of the plasma wake that is both accelerating and focusing for the positrons.

In simulations a $28 \mathrm{GeV}$ e beam, containing $1.8^{*} 10^{10}$ particles, is collided with a high-Z material target to generate $\mathrm{e}^{-}$and $\mathrm{e}^{+}$pairs. The initial positron distribution emerging from the $500 \mu \mathrm{m}$ Ta conversion target is generated using the Monte-Carlo code EGS, and is shown in Figure 1. The current profile of the $\mathrm{e}^{+}$beam roughly follows that of the incident $\mathrm{e}^{-}$beam, except that the number of produced $\mathrm{e}^{+}$is only $5 \%$ of the number of $\mathrm{e}^{-}$beam. The initial energy distribution of the $\mathrm{e}^{+}$beam is Maxwelllike with a $100 \%$ energy spread. The $\mathrm{e}^{-}$beam phase space is not affected much by the collisions with the target. We therefore neglect the effect of the target on the incident beam. We use a zero emittance $\mathrm{e}^{-}$beam while the emittance of the $\mathrm{e}^{+}$beam is calculated from the angle distribution plots.

The $\mathrm{e}^{-}$beam is used to ionize a $\mathrm{Li}$ vapor and drive a large amplitude plasma wave. The plasma electrons are expelled from the beam volume and return one-half plasma period later. The returning plasma electrons form a density concentration on axis behind the $\mathrm{e}^{-}$beam, leading to a large accelerating field for $\mathrm{e}^{-}$and $\mathrm{e}^{+}$particles in the back of the beam. The positrons located at the right phase are able to be trapped and injected into the plasma wave and thereafter to be accelerated.

\footnotetext{
CP877, Advanced Accelerator Concepts: $12^{\text {th }}$ Workshop, edited by M. Conde and C. Eyberger

(C) 2006 American Institute of Physics 978-0-7354-0378-9/06/\$23.00
} 

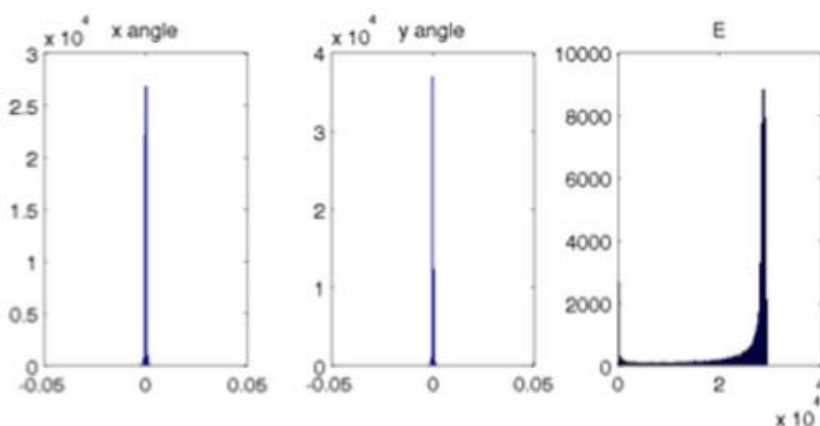

\section{Electrons}
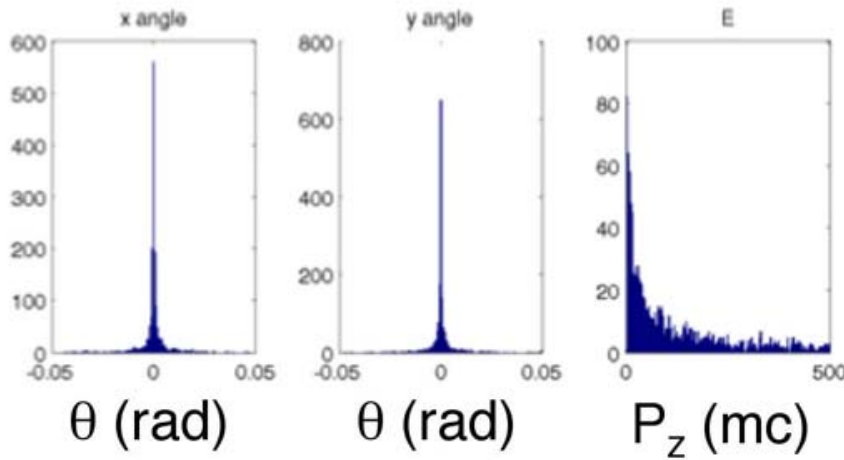

\section{Positrons}

FIGURE 1. EGS simulation results for the angle and energy distributions for $\mathrm{e}^{-}$beam (upper) and $\mathrm{e}^{+}$ beam (lower) after interaction with a $500 \mu \mathrm{m}$ tantalum target.

\section{SIMULATION PARAMETERS}

TABLE 1. Parameters for the 2-D cylindrical simulation of the plasma wake

\begin{tabular}{c|c}
\hline Bunch length $(\mu \mathrm{m})$ & 31.2 \\
\hline Spot Size $(\mu \mathrm{m})$ & 10 \\
\hline $\mathrm{e}^{-}$beam energy $(\mathrm{GeV})$ & 28 \\
\hline Drift momentum of $\mathrm{e}^{+}$beam $(\mathrm{mc})$ & $\mathrm{P}_{\mathrm{z}}=200 \quad \mathrm{P}_{\theta}=\mathrm{P}_{\varphi}=0$ \\
\hline Thermal momentum of $\mathrm{e}^{+}$beam $(\mathrm{mc})$ & $\mathrm{P}_{\mathrm{z}}=100 \quad \mathrm{P}_{\theta}=\mathrm{P}_{\varphi}=1$ \\
\hline Total number of e $\mathrm{e}^{-}$beam & $1.8^{*} 10^{10}$ \\
\hline Total number of $\mathrm{e}^{+}$beam & $9^{*} 10^{8}$ \\
\hline Plasma density $\left(\mathrm{cm}^{-3}\right)$ & $2.8^{*} 10^{17}$ \\
\hline Simulation box size $\left(\mathrm{c} / \omega_{\mathrm{p}}=23.725 \mathrm{um}\right)$ & $10^{*} 4$ \\
\hline Cells number & $600^{*} 200$ \\
\hline Grid size & $\mathrm{dz}=0.05, \mathrm{dr}=0.02$ \\
\hline $\mathrm{dt}\left(1 / \omega_{\mathrm{p}}\right)$ & .016 \\
\hline \# of beam particles per cell & 25 \\
\hline \# of gas particles per cell & 4 \\
\hline
\end{tabular}




\section{PRELIMINARY RESULTS}

Figure 2(a) shows the longitudinal wakefield $E_{1}$ generated by the $\mathrm{e}^{-}$beam. A positive field is accelerating for $\mathrm{e}^{+}$and decelerating for $\mathrm{e}^{-}$. The peak positive accelerating field (for $\mathrm{e}^{+}$) is $\approx 50 \mathrm{GV} / \mathrm{m}$, considerably higher than that generated by an $\mathrm{e}^{+}$beam with parameters similar to those of the $\mathrm{e}^{-}$beam. The region where the positrons are accelerated is very narrow and only a small portion of positrons generated by the $\mathrm{e}^{-}$beam can experience the accelerating and focusing field (near $\mathrm{z}=12.5 \mu \mathrm{m}$ in Figure 2(a) and Figure 2(c)). Figure 2(c) and 2(d) show that unlike in the electron blowout regime [1,2], the focusing force acting on the trapped $\mathrm{e}^{+}$bunch is neither constant along $\mathrm{z}$, nor linear with $\mathrm{r}$. This can lead to emittance growth of $\mathrm{e}^{+}$ bunch.
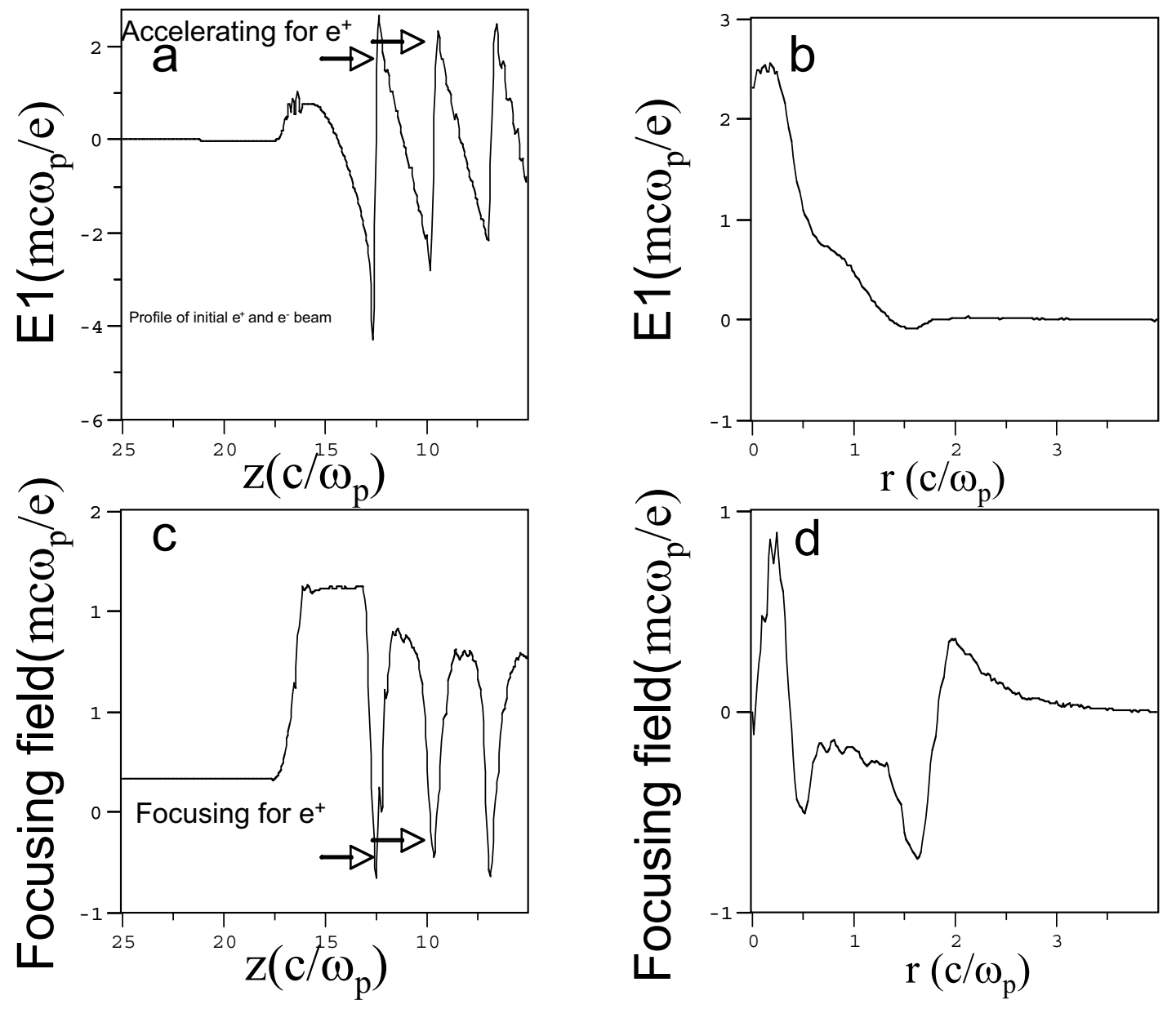

FIGURE 2. 2D simulation results at $\mathrm{s}=4.56 \mathrm{~cm}$ : (a) $\mathrm{E}_{1}$ lineout at $\mathrm{r}=0$ (on axis) (b) $\mathrm{E}_{1}$ lineout at $\mathrm{z}=12.3$ $\mathrm{c} / \omega_{\mathrm{p}}$ where the first $\mathrm{e}^{+}$bunch is located (c) focusing field lineout at $\mathrm{r}=0.5 \mathrm{c} / \omega_{\mathrm{p}}(\mathrm{d})$ focusing field lineout at $\mathrm{z}=12.3 \mathrm{c} / \omega_{\mathrm{p}}$. 


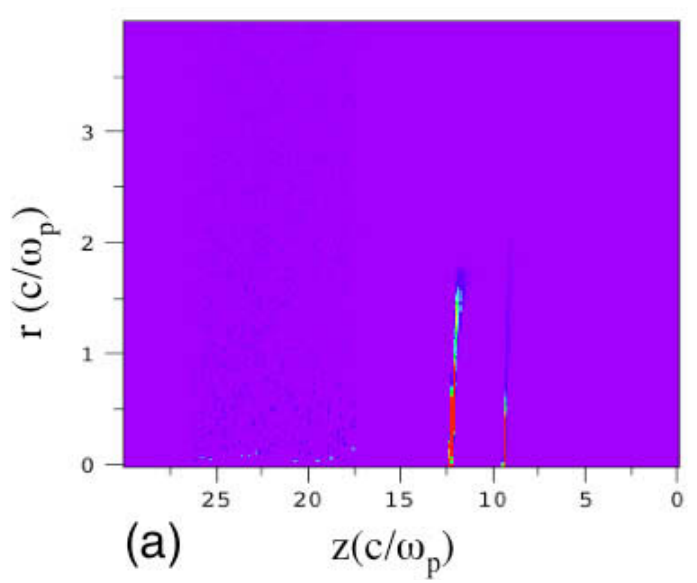

FIGURE 3. $2 \mathrm{D}$ simulation results at $\mathrm{s}=4.56 \mathrm{~cm}$ : (a) Phase space $\mathrm{P}_{\mathrm{z}}-\mathrm{z}$ of the $\mathrm{e}^{+}$bunches (b) Real space of the $\mathrm{e}^{+}$bunches.

The main body of the $\mathrm{e}^{+}$beam is expelled by the plasma ion column and cannot come back on axis and is lost. Figure 2(b) shows that the accelerating field depends on the radial position $r$. The positrons near the axis gain more energy than those faraway. The resulting continuous energy distribution for the two $\mathrm{e}^{+}$bunches is shown in Figure 3 (b).

Figure 3(a) shows that positrons are trapped and form two short bunches that are accelerated and focused. The length of the first $\mathrm{e}^{+}$bunch is $\approx 2.4 \mu \mathrm{m}(\mathrm{rms})$, comparable to that of $\mathrm{e}^{-}$bunch formed by trapping of plasma electrons observed in the E167 experiment[3]. The first $\mathrm{e}^{+}$bunch contains $1.78^{*} 10^{7}$ positrons, 10 times more than the second $\mathrm{e}^{+}$bunch. The maximum energy gain for first bunch is $\approx 1.75 \mathrm{GeV}$ over $\approx 4.56$ cm of plasma.

\section{CONCLUSION}

A new scheme for accelerating positrons in an $\mathrm{e}^{-}$beam driven plasma wake is investigated numerically. Preliminary simulation results show that two bunches of positrons are injected, focused, and accelerated to $\approx 1.75 \mathrm{GeV}$ over $\approx 4.56 \mathrm{~cm}$ of plasma.

The overlap of the focusing and accelerating phase of the plasma nonlinear wake for positrons is very small, and this limits the number of positrons that are trapped. On the other hand, it produces much shorter $\mathrm{e}^{+}$bunches. These may be of interest for light sources. The approach analyzed here provides a convenient means of studying physics issues for a possible $\mathrm{e}^{+}$plasma accelerator driven by an $\mathrm{e}^{-}$beam. The ultimate realization of this scheme would likely entail an injected short $\mathrm{e}^{+}$witness beam rather than the self-trapped case described here. 


\section{ACKNOWLEDGMENTS}

This work supported by U.S. Department of Energy Contract Nos. DE-FC0201ER41192, DE-AC02-76SF00515 (SLAC), DE-FG03- 92ER40745, DE-FG0398DP00211, DE-FG0392ER40727, and DE-AC-0376SF0098, and National Science Foundation Grant Nos. ECS-9632735, DMS-9722121, and PHY-0078715. Simulations were done at the USC Center for High Performance Computing and Communications (HPCC).

\section{REFERENCES}

1. J.B. Rosenzweig, B. Breizman, T. Katsouleas, and J.J. Su, Phys. Rev. A 44, 6189 (1991).

2. R. Fedele and U. de Angelis, Phys. Rev. A 33, 4412 (1986).

3. E. Oz, P. Muggli, T. Katsouleas et al., submitted to Physical Review Letters. 